\title{
Fabricating Tough Interpenetrating Network Cryogels with DNA as the Primary Network for Biomedical Applications
}

\author{
Sayantani Basu ${ }^{1}$, Rea Johl ${ }^{1}$, Settimio Pacelli², Stevin Gehrke ${ }^{1}$, Arghya Paul ${ }^{3 *}$ \\ ${ }^{1}$ Department of Chemical and Petroleum Engineering, School of Engineering, University of Kansas, Lawrence, Kansas, \\ USA \\ ${ }^{2}$ Department of Biomedical Engineering, The University of Texas at San Antonio, San Antonio, Texas, USA \\ ${ }^{3}$ Department of Chemical and Biochemical Engineering, Department of Chemistry, The University of Western Ontario, Lon- \\ don, ON N6A 5B9, Canada. \\ *E-mail: arghya.paul@uwo.ca
}

KEYWORDS. DNA, cryogel, interpenetrating network, alginate, toughness

1. Materials and Methods.

1.1. Formation of interpenetrating network DNA based hydrogels.

For the preparation of the hydrogels, sodium salt of DNA from salmon testes (Sigma-Aldrich, $\mathrm{M}_{\mathrm{n}} \approx 1.3 \times 10^{6} \mathrm{Da}$ ) was dissolved in Milli-Q water overnight at $35^{\circ} \mathrm{C}$. Chemical cross-linker, poly(ethylene glycol)diglycidyl ether (PEGDE, avg. $\mathrm{M}_{\mathrm{n}}=500$ ) along with catalyst, tetramethylethylenediamine (TEMED, $0.44 \% \mathrm{w} / \mathrm{v}$, Bio-Rad Laboratories Inc.) were subsequently added to the DNA. This mixture was continuously stirred with a magnetic stirrer for 5 minutes to ensure a homogenous mixing of the reactants. The reaction mixture was thereafter placed in well 24 plates or glass slides with rubber molds and kept at $-20^{\circ} \mathrm{C}$ for 48 hours. The concentration of DNA in the resultant hydrogel was fixed at $5 \%$ (w/v) and two different concentrations of the crosslinker were used. After the completion of reaction, the formed macroporous cryogels were thawed to room temperature before characterization. To generate the IPN hydrogels, the cryogels were soaked in $1 \%$ alginate $(\mathrm{w} / \mathrm{v})$ aqueous solution (sodium alginate, Mw $=$ $196000 \mathrm{~g} / \mathrm{mol}$ ) for 24 hours with gentle shaking at $37^{\circ} \mathrm{C}$. The alginate chains were subsequently crosslinked by immersing the hydrogels in different concentrations of $\mathrm{CaCl}_{2}$ such as $0.01 \mathrm{M}, 0.025 \mathrm{M}, 0.05 \mathrm{M}, 0.1 \mathrm{M}$ and $0.2 \mathrm{M}$. For comparison as control groups, the conventional hydrogels were formed at $55^{\circ} \mathrm{C}$ following previously published protocols ${ }^{1}$.

1.2. Mechanical characterization of the hydrogels.

The response of the single and double network hydrogels to shear forces were studied using an AR2000 rheometer (TA Instruments, New Castle, DE). Single network cryogels were thawed to room temperature soaked in 1X PBS (Phosphate Buffer Saline) overnight prior to rheological testing. All the tests were performed at $37^{\circ} \mathrm{C}$ using $20 \mathrm{~mm}$ rough parallel plate and plate geometry. The linear viscoelastic region was determined by performing strain sweep by varying strain percentage values from 0.1 to 10,000 , at a constant frequency of $1 \mathrm{~Hz}$. The frequency and stress sweep monitoring the storage and loss moduli were carried out in the linear viscoelastic region at $1 \%$ strain. Uniaxial compression tests were performed using RSA3 dynamic mechanical analyzer (TA Instruments, New Castle, DE). All the experiments were conducted with pre-soaked (24h in 1X PBS) gels and cylindrical gel samples of $4 \mathrm{~mm}$ diameter and $3-4 \mathrm{~mm}$ thickness. The compressive modulus of the gel (compressed at $0.005 \mathrm{~mm} / \mathrm{s}$ strain rate) was determined from the slope of the stress-strain curve in the region between $10 \%-20 \%$ strain. At high strains the water entrapped in the cryogel constructs was observed to be exuded, thus making it possible to go beyond $0.9 \mathrm{~mm} / \mathrm{mm}$ strain during the measurements. The toughness was calculated by determining the area under the stress-strain curve, i.e the work to fracture. For a better insight into the energy dissipation, hysteresis experiments were performed where three cycles of loading and unloading were applied to the hydrogels to a strain of $0.4 \mathrm{~mm} / \mathrm{mm}$. The area enclosed within the loading and unloading plot was designated as the energy dissipated.

1.3. Physical, chemical and morphological characterization of the hydrogels.

Swelling experiments were performed to understand the water uptake capacity of the gel. Freeze dried gels with known initial mass $\left(\mathrm{M}_{0}\right)$ were soaked in 1X PBS. The mass of the swollen gel was noted at every specified timepoint $\left(\mathrm{M}_{t}\right)$ and the swelling ratio was determined by the following equation,

$$
\text { swelling ratio }=\frac{M_{t}-M_{0}}{M_{0}}
$$

Finally, the morphology of the single and double network hydrogels was visualized by performing scanning electron microscopy (SEM) with the freeze-dried hydrogels after sputter coating the samples with gold. An immersion-lens detector (FEI Tecnai F20 XT) with an acceleration voltage of $1-10 \mathrm{kV}$ was used for acquiring the images. The pore diameter of the hydrogels was evaluated from the SEM images using Image J software. 50 measurements from five different images captured from different sections of the hydrogel were used to measure the pore diameter values for each group. Energy dispersive X-ray spectroscopy was carried out with freeze dried hydrogels for the detection of the elemental composition of the hydrogel formulations.

1.4. Analysis of the in vitro biocompatibility of the hydrogels.

Human adipose-derived stem cells (hASCs, RoosterBio, USA). hASCs from passage 3 to 5 were grown in Invitrogen $\alpha$-minimum essential medium $(\alpha-\mathrm{MEM})$ with $10 \%$ fetal bovine serum (FBS, Thermo-Fischer Scientific, USA) and $1 \%$ of penicillin/streptomycin in an incubator $\left(37^{\circ} \mathrm{C}, 5 \% \mathrm{CO}_{2}\right)$ for the in vitro biocompatibility studies. This cell line was selected for our studies due to their easy harvesting procedure and high proliferation rates ${ }^{2}$ ${ }^{3}$. hASCs were seeded in a 24 well plate, and the respective hydrogels were placed inside a cell culture insert (Corning Incorporated - Life Sciences, $3 \mu \mathrm{m}$ pore diameter). After respective time intervals ( $24 \mathrm{~h}$ and $72 \mathrm{~h}$ ) MTS assay (Promega, USA) was performed to demonstrate the cell viability. 
Additionally, a live/dead assay was carried out to image live/dead cells after $24 \mathrm{~h}$ and $72 \mathrm{~h}$ of contact with the hydrogels following experimental procedures as previously published ${ }^{1}$.

1.5. Statistical Analysis. For the statistical analysis one-way analysis of variance (ANOVA) was performed followed by Tukey's multiple comparison tests. Two tailed unpaired t test was performed for comparison of two experimental groups. GraphPad Prism 5 was used for all the statistical analyses.
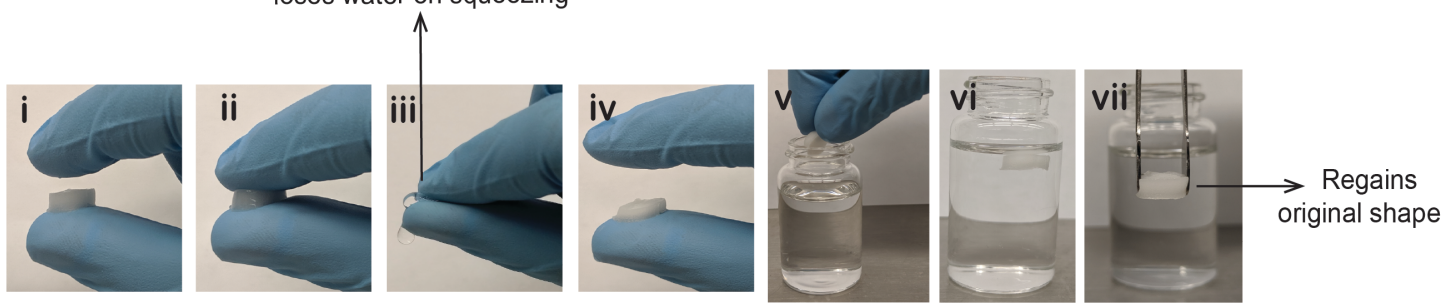

Figure S1. Behavior of the IPN cryogels under compressive forces. Images marked as (i) - (vii) display the behavior of the hydrogels while compressing it and thereafter re-swelling in water after the removal of compressive forces.

(a)

\begin{tabular}{|l|l|}
\hline $\begin{array}{l}\text { Different conc. of } \\
\text { PEGDE }\end{array}$ & $\begin{array}{l}\text { Mass ratio of } \\
\text { DNA/PEGDE }\end{array}$ \\
\hline Low & $5 / 2.5=2$ \\
\hline High & $5 / 3.5=1.43$ \\
\hline
\end{tabular}

(b)

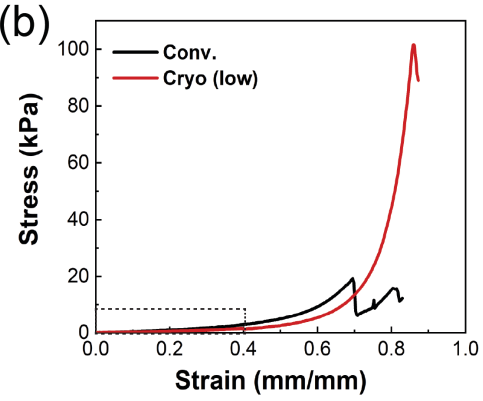

(c)

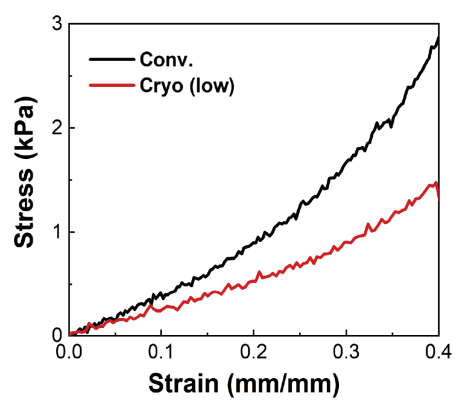

(d)

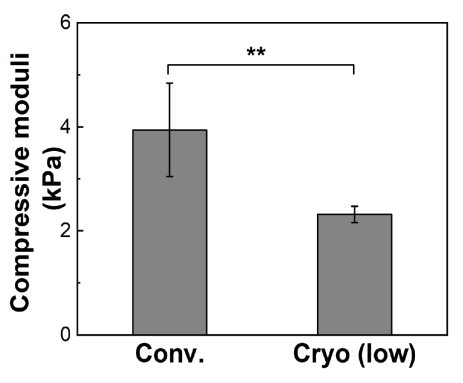

Figure S2. Mechanical characterization of the single network cryogels. (a) Nomenclature of the hydrogels with different concentrations of the crosslinker. (b) Stress vs strain plot for the normal and cryogels formulated with low concentration of the crosslinker, PEGDE under compressive forces. (c) Magnification of the stress vs strain plots in the region of $0-0.4 \mathrm{~mm} / \mathrm{mm}$ strain. (d) Comparison of the compressive moduli of the normal and cryogels, estimated from the slope of the stress vs strain plot in the region of $0.1-0.2 \mathrm{~mm} / \mathrm{mm}$ strain. Results are represented as mean \pm standard deviation $(n=5)(* * p<0.01)$

(a)

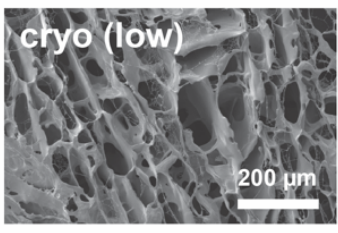

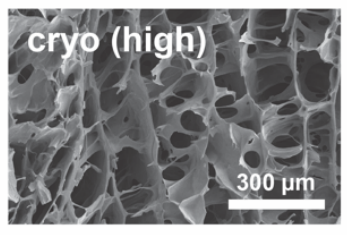

(b)

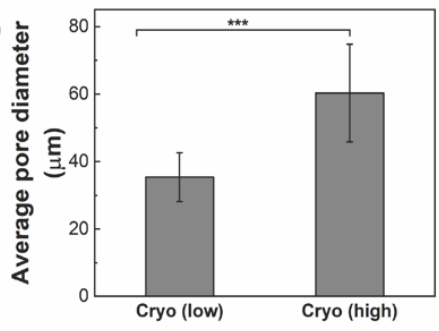


Figure S3. Morphological characterization of the single network cryogels. (a) Scanning electron microscope images of the single network cryogels with different concentrations (low and high) of the crosslinker. (b) Comparison of pore size of the formulated cryogels with low and high concentration of the crosslinker.

(a)

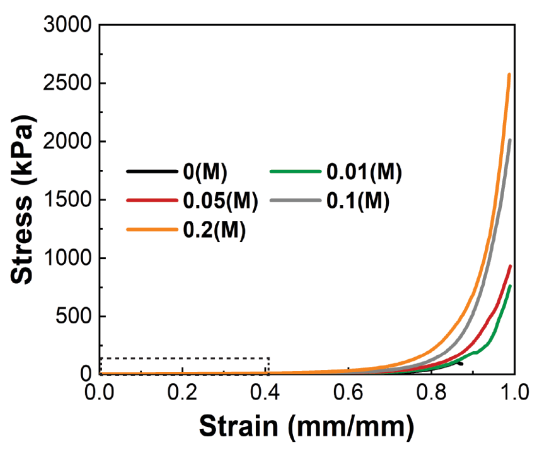

(b)

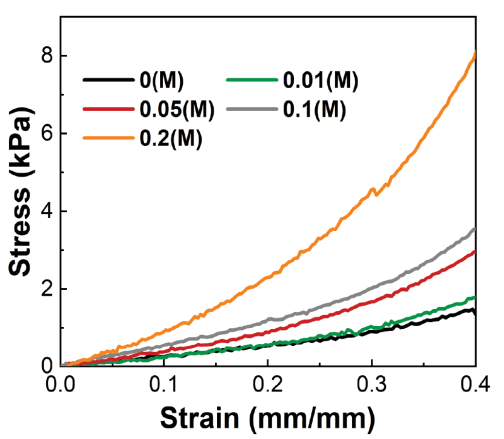

(c)

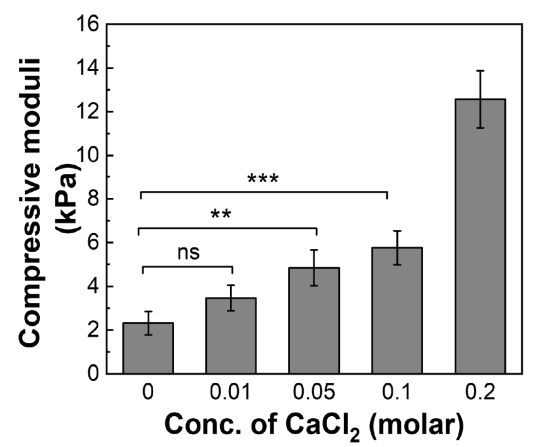

Figure S4. Mechanical characterization of the IPN cryogels formulated with cryo (low) as the primary network. (a) Stress vs strain plots of the IPN cryogels as a function of calcium chloride concentration from $0 \mathrm{M}$ to $0.2 \mathrm{M}$. The group designated as $0 \mathrm{M}$ represents the single network cryogels with lower concentration of the crosslinker (Mass ratio of DNA /PEGDE $=2$ ) whereas the other groups represent the IPN cryogels and were labelled with the concentration of calcium chloride used for the IPN network formation. (b) Magnified section of the stress vs strain plot upto $0.4 \mathrm{~mm} / \mathrm{mm}$ strain. (c) Comparison of the compressive moduli of IPN hydrogels with increasing concentration of calcium chloride. The moduli values were estimated from the slope of the stress vs strain plot in the region of $0.1-0.2 \mathrm{~mm} / \mathrm{mm}$ strain. The nomenclature of the groups is consistent with the ones from the stress vs strain plots. Results are shown as mean \pm standard deviation ( $\mathrm{n}=5$ ) $(* * \mathrm{p}<0.01, * * * \mathrm{p}<0.001)$. 
(a)
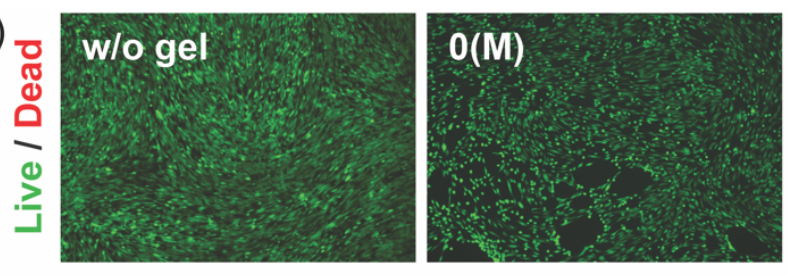

(b)
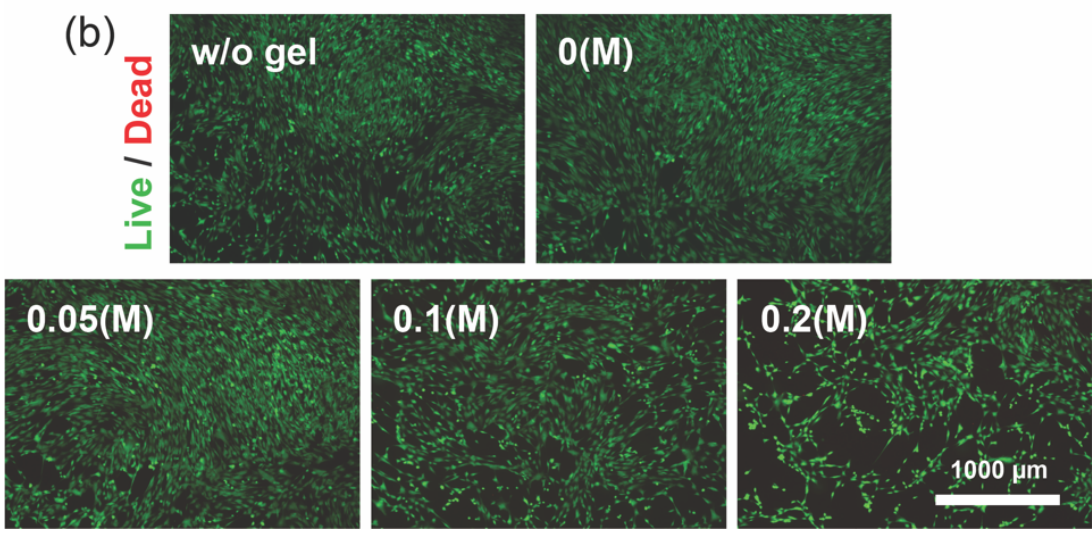

Figure S5. Characterization of the in vitro cytotoxicity of the formulated hydrogels. (a) Fluorescent images of hASCs after live-dead assay at 72 hours timepoint. (b) Fluorescent images of hASCs after live-dead assay at 24 hours timepoint. The group designated as $0 \mathrm{M}$ represents the single network cryogels with higher concentration of the crosslinker (Mass ratio of DNA /PEGDE $=1.43$ ) whereas the other groups represent the IPN cryogels and were labelled with the concentration of calcium chloride used for the IPN network formation.

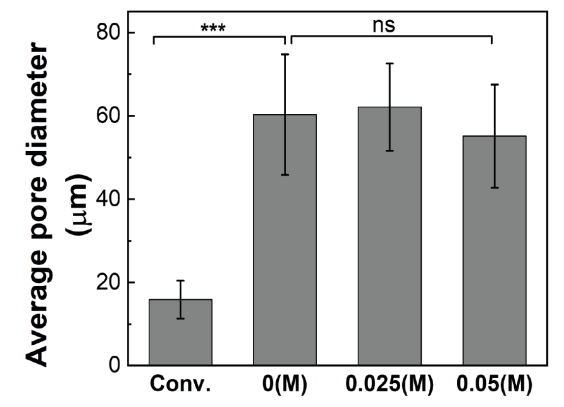

Figure S6. Comparison of pore size (from the SEM images) of the formulated hydrogels developed via conventional and cryo technique with higher concentration of the crosslinker (Mass ratio of DNA /PEGDE $=1.43$ ) along with the IPN cryogels $(* * * p<0.001)$.

(a)
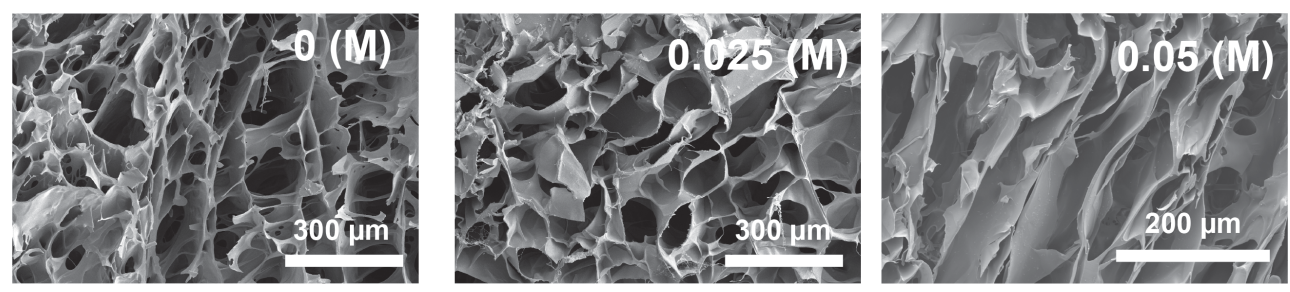

Figure S7. Morphological characterization of the single and IPN cryogels formulated with mass ratio of DNA /PEGDE $=1.43$ (a) Crosssectional SEM images of Cryo-SN and Cryo-IPN. 0(M) denotes the single network cryogels whereas $0.025(\mathrm{M})$ and $0.05(\mathrm{M})$ are designated to IPN hydrogels formed with $0.025 \mathrm{M}$ and $0.05 \mathrm{M} \mathrm{CaCl}_{2}$. 
(a)

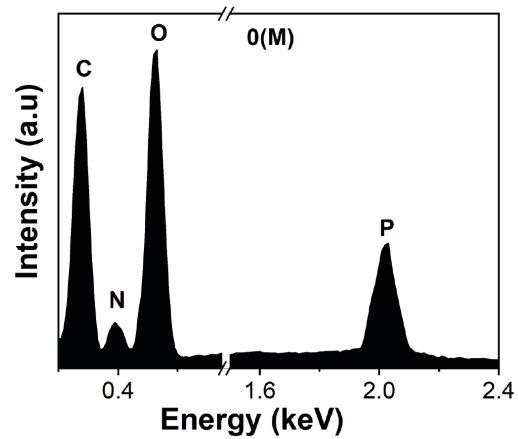

(b)

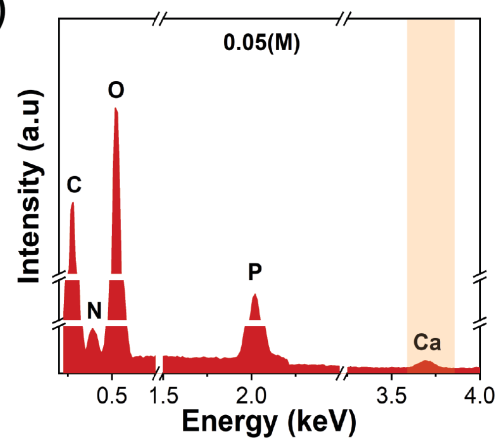

Figure S8. (a) Energy-dispersive X-ray spectra of the single network cryo (high). (b) Energy-dispersive X-ray spectra of IPN hydrogels formed with $0.05 \mathrm{M}$ calcium chloride. The presence of the peak corresponding to calcium is highlighted in the spectra.

(a)

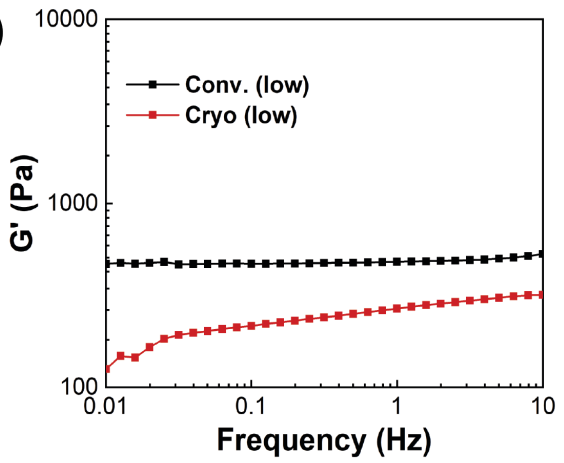

(b)

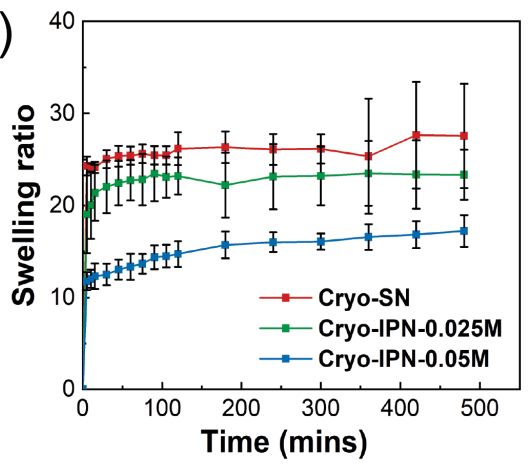

Figure S9. Characterization of rheological and physical properties of the formulated hydrogels. (a) Comparison of frequency sweep results of the hydrogels formed via conventional and cryo methods with low concentration of the crosslinker PEGDE. (b) Effect of $\mathrm{Ca}^{2+}$ concentration on the swelling ratio of the hydrogels. Swelling ratio is plotted as a function of time for the single (Cryo-SN) and IPN cryogels developed with high concentration of the crosslinker PEGDE. Cryo-IPN-0.025M and Cryo-IPN-0.05M designate IPN hydrogels made with $0.025 \mathrm{M}$ and $0.05 \mathrm{M} \mathrm{CaCl}_{2}$ respectively.

\section{References}

1.Basu, S.; Alkiswani, A.-R.; Pacelli, S.; Paul, A., Nucleic Acid-Based Dual Cross-Linked Hydrogels for in Situ Tissue Repair via Directional Stem Cell Migration. ACS Applied Materials \& Interfaces 2019, 11 (38), 34621-34633.

2.Pacelli, S.; Maloney, R.; Chakravarti, A. R.; Whitlow, J.; Basu, S.; Modaresi, S.; Gehrke, S.; Paul, A., Controlling Adult Stem Cell Behavior Using Nanodiamond-Reinforced Hydrogel: Implication in Bone Regeneration Therapy. Scientific Reports 2017, 7 (1), 6577.

3.Pacelli, S.; Basu, S.; Berkland, C.; Wang, J.; Paul, A., Design of a Cytocompatible Hydrogel Coating to Modulate Properties of Ceramic-Based Scaffolds for Bone Repair. Cellular and Molecular Bioengineering 2018, 11 (3), 211-217. 